\title{
Ring chromosome formation as a novel escape mechanism in patients with inverted duplication and terminal deletion
}

\author{
Jeroen Knijnenburg ${ }^{1}$, Arie van Haeringen ${ }^{2}$, Kerstin BM Hansson ${ }^{2}$, Arjan Lankester ${ }^{3}$, \\ Margot JM Smit ${ }^{4}$, René DM Belfroid ${ }^{2}$, Egbert Bakker ${ }^{2}$, Carla Rosenberg ${ }^{5}$, Hans J Tanke ${ }^{1}$ and \\ Károly Szuhai*,1
}

\footnotetext{
${ }^{1}$ Department of Molecular Cell Biology, Leiden University Medical Center, Leiden, The Netherlands; ${ }^{2}$ Department of Clinical Genetics, Leiden University Medical Center, Leiden, The Netherlands; ${ }^{3}$ Department of Pediatrics, Leiden University Medical Center, Leiden, The Netherlands; ${ }^{4}$ Location Juliana Kinderziekenhuis, Hagaziekenhuis, Den Haag, The Netherlands; ${ }^{5}$ Department of Genetics and Evolutionary Biology, Institute of Biosciences, University of São Paulo, São Paulo, Brazil
}

Ring chromosomes are rare cytogenetic findings and are associated at phenotypic level with mental retardation and congenital abnormalities. Features specific for ring chromosome syndromes often overlap with the features of terminal deletions for the corresponding chromosomes. Here, we report a case of a ring chromosome 14 which was identified by conventional cytogenetics and shown to have a terminal deletion and an additional inverted duplication with a triplication by using large insert clone and oligo array-comparative genomic hybridization (array-CGH), fluorescence in situ hybridization (FISH) and multiplex ligation-dependent probe amplification (MLPA). The combination of an inverted duplication with a terminal deletion in a ring chromosome is of special interest for the described syndromes of chromosome 14. The presented findings might explain partly overlapping clinical features described in terminal deletion, duplication and ring chromosome 14 cases, as these rearrangements can be easily overlooked when performing GTG-banding only. Furthermore, we suggest that ring chromosome formation can act as an alternative chromosome rescue next to telomere healing and capture, particularly for acrocentric chromosomes. To our knowledge, this is the first time an inverted duplication with a terminal deletion in a ring chromosome is identified and characterized using high-resolution molecular karyotyping. Systematic evaluation of ring chromosomes by array-CGH might be especially useful in distinguishing cases with a duplication/deletion from those with a deletion only. European Journal of Human Genetics (2007) 15, 548-555. doi:10.1038/sj.ejhg.5201807; published online 7 March 2007

Keywords: ring chromosome; array-CGH; inverted duplication

${ }^{*}$ Correspondence: Dr K Szuhai, Department of Molecular Cell Biology, Leiden University Medical Center, PO Box 9600, 2300 RC Leiden, zone S-1-P, The Netherlands.

Tel: + 3171 5269211; Fax: + 3171 5268270;

E-mail: k.szuhai@lumc.nl

Received 16 August 2006; revised 31 January 2007; accepted 7 February 2007; published online 7 March 2007
Introduction

Ring chromosomes are uncommon findings in pre- and post-natal cytogenetics. Inheritance of ring chromosomes has been reported ${ }^{1-3}$ but the majority of ring chromosomes are de novo. They have been reported for all human chromosomes and are known to cause multiple congenital anomalies and mental retardation. Ring chromosomes are generally believed to result from distal breakage of the 
short and long arm of a chromosome and rejoining of the ends. ${ }^{4}$ There are also reports with no apparent deletion of the telomeric ends, thus resulting in complete ring chromosomes. ${ }^{5}$ Patients with ring chromosomes often exhibit a general overlap in phenotype, which has coined the term 'ring syndrome'. ${ }^{6}$ Growth failure with no or minor anomalies is found to be the major abnormality in patients with complete ring chromosomes and is thought to be the result of cell death during development. Indeed, sister chromatid exchange during the cell cycle may cause mechanical interference of the cell division because of disruption, breakage, entangling or doubling of rings, resulting in aneuploidy and possible death of the daughter cells. In a study of 207 patients carrying a ring chromosome, ${ }^{6}$ one-fifth showed to be affected with this general ring syndrome. When both telomeric ends and coding sequences are deleted, the phenotype of the patient is in general more severe, often with specific characteristics related to the chromosome involved.

Here we report the characterization of a ring chromosome 14 containing a terminal deletion and an inverted duplication with a triplication by using molecular cytogenetic tools such as oligo array-comparative genomic hybridization (array-CGH), multiplex ligation-dependent probe amplification (MLPA) and fluorescence in situ hybridization (FISH). The existence of duplication with a terminal deletion in a ring chromosome similar to other duplication/deletion cases ${ }^{7,8}$ might have clinical consequences in patients with ring chromosome 14 syndrome. This finding might explain the overlapping clinical features in patients with a ring chromosome 14 compared with patients with a terminal duplication of chromosome 14, as existing duplications in ring chromosomes can be easily overlooked at the cytogenetic level. Accordingly, the large overlap in clinical features between published patients with a distal duplication and those with a distal deletion suggests that some duplication patients have an accompanying distal deletion, similar to the patient discussed by Chen et al. ${ }^{9}$

Here we show the importance of using combined molecular cytogenetic techniques in the characterization of chromosomal alterations, in particular in patients with ring chromosomes.

\section{Case report}

The proband is an 8-year-old girl of healthy, nonconsanguineous parents. She has two sisters and two brothers, all normal. Ultrasound examination at 34 weeks of gestation showed generalized growth retardation and one umbilical artery. Birth was at 37 weeks, birth weight was $1970 \mathrm{~g}$ (p30) and head circumference was $30.5 \mathrm{~cm}(<\mathrm{p} 3)$.

Examination at 4 months revealed craniofacial dysmorphisms, namely hypertelorism, upturned nose, broad nasal bridge, malformed helices and mild micrognathia. A single palmar crease bilaterally and abnormally implanted toes were noted. At 9 months; a patent ductus arteriosus was surgically closed. At present, she is severely developmentally delayed. She is microcephalic $(<\mathrm{p} 3)$, has short stature $(<\mathrm{p} 3)$ and low weight $(<\mathrm{p} 3)$. Growth hormone $(\mathrm{GH})$ and insulin-like growth factor 1 (IGF1) levels were normal. She suffers from recurrent upper and lower airway infections, eczema, scoliosis and retinitis pigmentosa. She has hypogammaglobulinemia (IgG/M and A) and normal numbers of peripheral B lymphocytes. She has sleeping difficulties, poor feeding and seizures. Informed consent was obtained from parents and patient according to routine LUMC procedure.

\section{Materials and methods}

Conventional cytogenetic analysis on GTG-banded chromosomes from cultured lymphocytes of the patient and the parents was performed according to standard techniques. From the proband and the parents, 100 and 50 cells were analyzed, respectively.

FISH was performed according to standard protocols on metaphase chromosomes or interphase nuclei of the proband, using Cy5-ULS or D-Green-ULS labeled Whole Chromosome Painting probe (WCP) no. 14 (Kreatech Biotechnology, Amsterdam, The Netherlands), half-YAC clone yRM2006 and Vysis ${ }^{\circledR}$ LSI $^{\circledR}$ IGH/CCND1 combined probe (for 11q13 and 14q32.33, respectively) (Abbott Molecular, Hoofddorp, The Netherlands). Three BAC clones that mapped at $14 \mathrm{q} 32.12$ and two at $14 \mathrm{q} 32.33$, namely RP11-258D14, RP11-489D22, RP11-371E8, RP1173M18 and RP11-417P24, respectively (Table 1), labeled with Cy3-dUTP (GE Healthcare, Diegem, Belgium) or FITCdUTP (Roche diagnostics, Almere, The Netherlands) were used for further confirmations.

Array-CGH was performed using $\sim 1.0 \mathrm{Mb}$ spaced wholegenome large insert clone arrays, which were made available by the Wellcome Trust Sanger Institute (http:// www.sanger.ac.uk). The clones were grown, amplified and spotted as described previously. ${ }^{10,11}$ Genomic DNA of the patient was isolated using standard techniques, and $500 \mathrm{ng}$ was labeled with Cy3-dCTP (GE Healthcare, Diegem, Belgium) using the BioPrime ${ }^{\circledR}$ DNA Labeling System (Invitrogen, Breda, The Netherlands). As a reference DNA, $500 \mathrm{ng}$ female human genomic DNA (Promega, Leiden, The Netherlands) was labeled using Cy5-dCTP. Hybridization and slide washing was performed without prehybridization on a HS400 hybridization station (Tecan, Giessen, the Netherlands). The arrays were scanned with a GenePix 4100A scanner (Axon Instruments, Union City, CA, USA) and the images were processed using GenePix Pro 4.1 software. Final analysis of the intensity ratios of the hybridized DNA was preformed using Microsoft Excel according to published standards. ${ }^{10}$ 
Table 1 Genomic location of BAC clones used in confirmatory FISH and genomic location of oligos around the breakpoints found with oligo array-CGH

\begin{tabular}{llcl}
\hline BAC clone & Locus & Region $^{\text {a }}$ & Involvement $^{\text {In proximal side of triplication }}$ \\
\hline RP11-258D14 & $14 q 32.12$ & $92079320-92288476$ & In distal side of triplication \\
RP11-489D22 & $14 q 32.12$ & $92428426-92630687$ & In proximal side of duplication \\
RP11-371E8 & $14 q 32.13$ & $92565641-92758891$ & In distal side of duplication \\
RP11-73M18 & $14 q 32.33$ & $103217347-103382885$ & In proximal side of deletion \\
RP11-417P24 & $14 q 32.33$ & $105267358-105437117$ & \\
Oligo & Locus & Region & Involvement \\
A_14_P101212 & $14 q 32.12$ & $91679319-91679378$ & In proximal side of triplication \\
A_16_P02972545 & $14 q 32.12$ & $92360710-92360769$ & In distal side of triplication \\
A_14_P109278 & $14 q 32.12$ & $92371629-92371688$ & In proximal side of duplication \\
A_16_P02989318 & $14 q 32.33$ & $103716114-103716166$ & In distal side of duplication \\
A_16_P02989342 & $14 q 32.33$ & $103727207-103727260$ & In proximal side of deletion \\
\hline
\end{tabular}

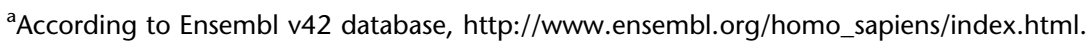

For further high-resolution analysis of duplications and deletions, oligo array-CGH was performed using the Agilent Human Genome CGH Microarray Kit 244K (Agilent, Amstelveen, The Netherlands) according to the manufacturer's instructions following protocol version 4.0. Data analysis was performed with the CGH Analytics 3.4 software platform (Agilent, Amstelveen, The Netherlands).

MLPA was performed as described by White et al. ${ }^{12}$ The selected probes were ordered from Invitrogen (Breda, the Netherlands), sequences are available as online Supplementary Data. Quantitative readout was carried out using the ABI 3730 DNA analyzer (Applied Biosystems, Nieuwerkerk a/d IJssel, The Netherlands). The accompanying Genescan 3.5 software was used for peak analysis and further downstream normalization and calculations were performed in Microsoft Excel as described before. ${ }^{12}$

Quantitative fluorescent polymerase chain reaction (QF-PCR) was performed using polymorphic short tandem repeat (STR) markers for allelotyping. Markers were chosen to cover the distal part of chromosome $14 \mathrm{q}$, from $14 \mathrm{q} 24.2$ to 14 qter. ${ }^{9}$

\section{Results}

Cytogenetic analysis resulted in a ring chromosome 14 in 95 of the 100 analyzed cells from the patient (Figure 1a), whereas the remaining five cells showed a complex rearrangement involving endoreduplication of the ring chromosome. Both parents presented a normal karyotype. FISH using the yRM2006 probe demonstrated the absence of the subtelomeric region of $14 \mathrm{q}$ in the ring chromosome. In a later stage, when the proband presented with recurrent infections, the deletion was shown to encompass the $\operatorname{IgH}$ gene using the $\mathrm{LSI}^{\circledR}$ IGH/CCND1 combined probe, which is located at $14 \mathrm{q} 32.33$ at about $1 \mathrm{Mb}$ from the telomere (data not shown).

Array-CGH was performed to further map the deletion. The result revealed an additional duplication of $14 q 32.12$
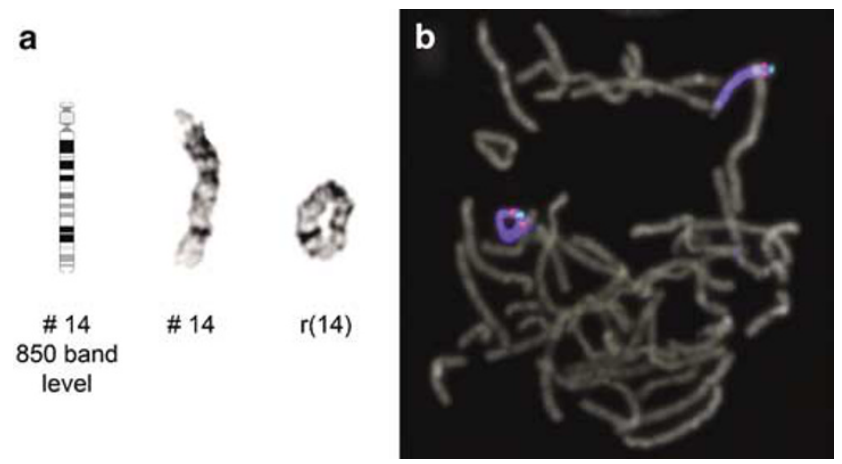

Figure 1 The ring chromosome 14 of the patient and its normal homolog. (a) GTG-banded images (b). FISH applied to a metaphase cell with BAC RP11-73M18 (red), BAC RP11-371E8 (green) and whole chromosome paint for chromosome 14 (blue), showing the inverted duplication in the ring chromosome.

to $14 \mathrm{q} 32.32$ (Figure 2), next to the already detected deletion of $14 \mathrm{q} 32.33$ to 14 qter. The size of the duplication was found to be $10.8-13.5 \mathrm{Mb}$, from BAC clone RP11$73 \mathrm{M} 18$ to RP11-371E8, whereas the size of the deletion was $1.1-3.0 \mathrm{Mb}$, from BAC clone RP11-417P24 to CTC$820 \mathrm{M} 16$. These findings were confirmed by FISH, whereby the duplicated clones were chosen most proximal (RP1173M18) and distal (RP11-371E8) to the centromere. The FISH showed the duplication to be inverted (Figure 1b). BAC clone RP11-417P24, located within the deleted area, was used to confirm the deletion (data not shown). FISH using the same BAC probe sets showed no alteration (gain, loss or inversion) in any of the parents.

Analysis with polymorphic markers that map to the deletion, duplication and a normal part of the chromosome, proved the duplication to be intrachromosomal. The marker analysis showed a duplication of one allele of markers D14S557 and D14S543 (Table 2). Parental analysis revealed that the ring chromosome is of paternal origin.

Additional high-resolution oligo array-CGH confirmed the previous findings, the size of the amplified region was 
estimated to be $11.344 \mathrm{Mb}$ and the size of the deletion was estimated to be $2.641 \mathrm{Mb}$. The genomic locations of the oligos around the breakpoint are found in Table 1 . Interestingly, this assay revealed a triplicated $681 \mathrm{~kb}$ region in the proximal duplication region, belonging to band 14q32.12 (Figure 3). A confirmatory MLPA test using probes designed for all altered regions (triplicated, duplicated, deleted and normal) proved the presence of all alterations, including the triplication of the region in band 14q32.12 (Figure 4) and it showed that the parents of the proband have a normal copy number for all tested regions. FISH using BAC's RP11-258D14 and RP11-489D22 showed that the triplicated region is located within the ring, at the distal side of the rearranged q-arm (data not shown).

\section{Discussion}

Using the combination of FISH and array-CGH in genetic analysis has demonstrated that seemingly simple rearrangements are often more complex than defined by
GTG-banding. ${ }^{10,13,14}$ After conventional cytogenetic screening (Figure 1a), the karyotype of the proband was described as 46,XX,r(14)(p12q32). To further map the breakpoint, array-CGH was performed with a resolution of about $1 \mathrm{Mb}$ (Figure 2). Next to the expected deletion of band $14 \mathrm{q} 32.33$ an additional duplication of $14 \mathrm{q} 32.12$ to $14 q 32.32$ was revealed. FISH using the first and, the last duplicated BAC clone confirmed this finding and showed the duplication to be inverted (Figure 1b), which originated from one paternal chromosome based on STRallelotyping (Table 1).

An inverted duplication associated with a terminal deletion was first described for distal chromosome $8 p,^{7,8}$ followed by $1 \mathrm{q},{ }^{15} 4 \mathrm{q},{ }^{16} 15 \mathrm{q},{ }^{17}$ and more recently, also for chromosome 14q. ${ }^{9}$ A mechanism for the formation of inverted duplications associated with terminal deletions has been described both for intrachromosomal duplications, based on normal parental chromosomes ${ }^{18}$ and for intra- and interchromosomal duplications based on a parental inversion carrier. ${ }^{19}$ These proposed mechanisms

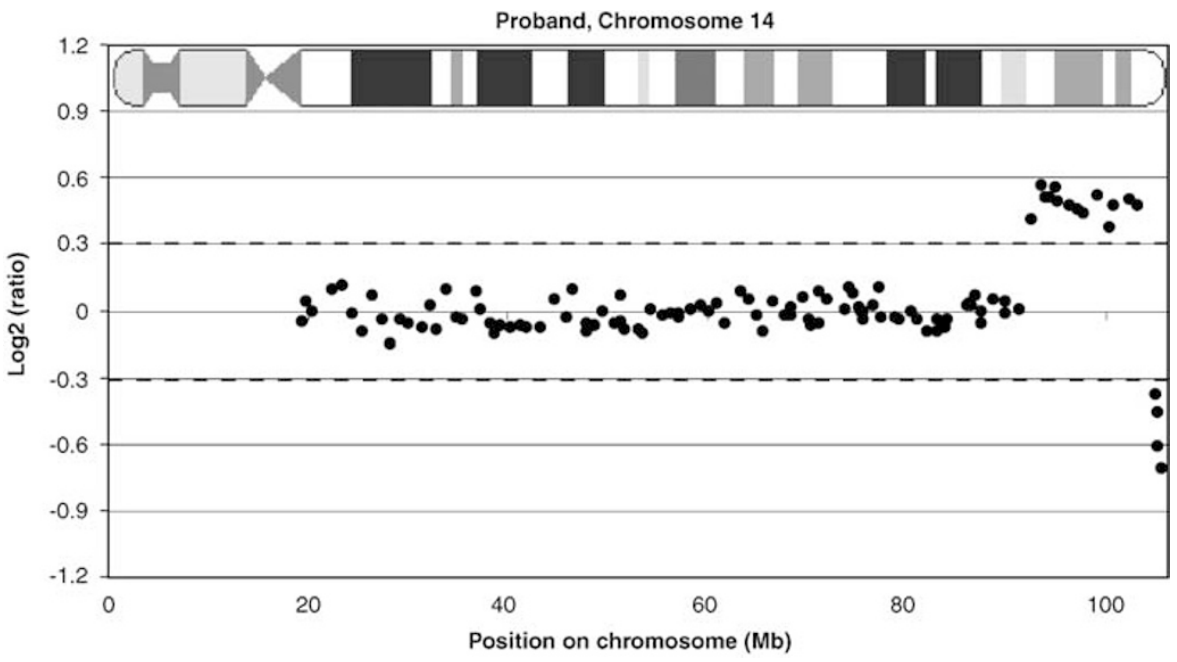

Figure 2 Array-CGH log 2 ratio plot of chromosome 14 of the proband at about $1 \mathrm{Mb}$ resolution, showing a distal duplication and a terminal deletion of chromosome 14 material. The dotted horizontal line at -0.3 and 0.3 represents the threshold line for deletions and duplications, respectively.

Table 2 Location of STR markers and genotypic results in the proband and her parents

\begin{tabular}{llcrrr}
\hline Markers & Locus & Region $^{\text {a }}$ & Mother & Father & Proband \\
\hline D14S620 & $14 q 24.2$ & $72401116-72401231$ & 114,114 & 114,118 & 114,118 \\
D14S739 & $14 q 31.1$ & $81336367-81336556$ & 184,184 & 176,184 & 176,184 \\
D14S616 & $14 q 31.3$ & $84263655-84263874$ & 211,215 & 223,223 & 211,223 \\
D14S128 & $14 q 31.3$ & $85450372-85450704$ & 336,363 & 332,344 & 332,336 \\
D14S617 & $14 q 32.12$ & $91272543-91272683$ & 139,161 & 161,165 & 139,165 \\
D14S557 & $14 q 32.32$ & $102189847-102190162$ & 304,320 & 275,287 & $287,287,304$ \\
D14S543 & $14 q 32.33$ & $103658598-103658852$ & 244,252 & 241,249 & $249,249,252$ \\
\hline
\end{tabular}

${ }^{a}$ According to Ensembl v42 database, http://www.ensembl.org/homo_sapiens/index.html. 
Proband, 14q32.11 to telomere

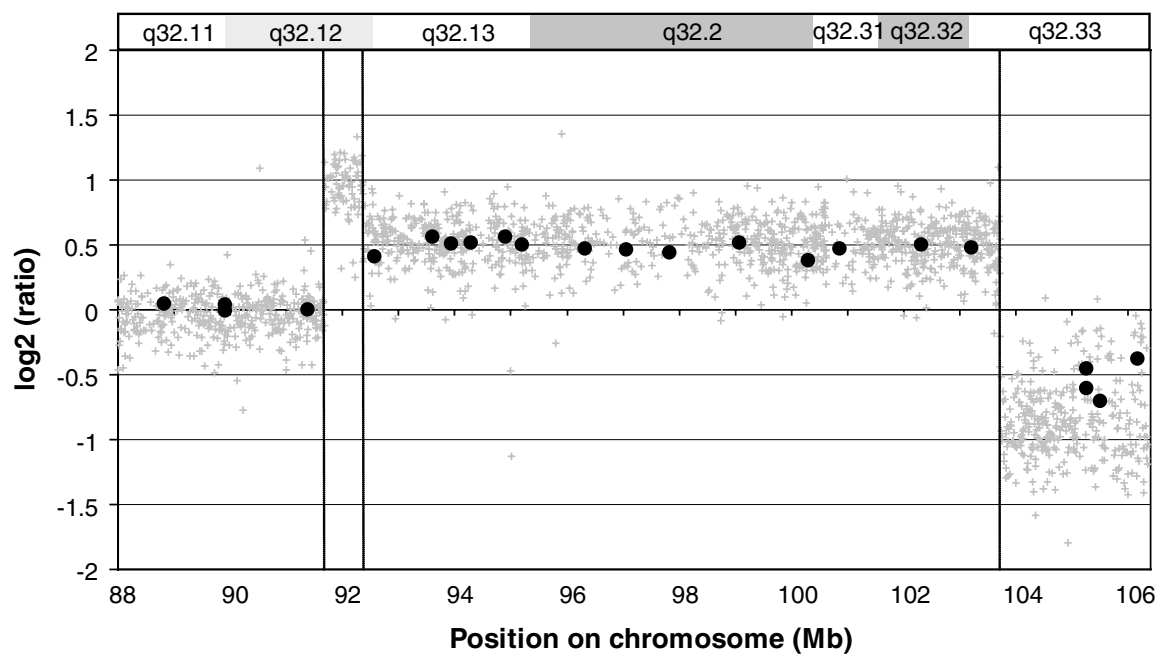

Figure 3 A combined display of the oligo array-CGH (open circles) and the $\pm 1 \mathrm{Mb}$ resolution large insert clone array-CGH (closed circles), log 2 ratio plots of the chromosome 14q32.11-qter region of both experiments on the proband are shown. The oligo array shows a triplication, a duplication and a deletion of the distal region of chromosome 14 . The clones of the $1 \mathrm{Mb}$ array fall just adjacent to the triplication.

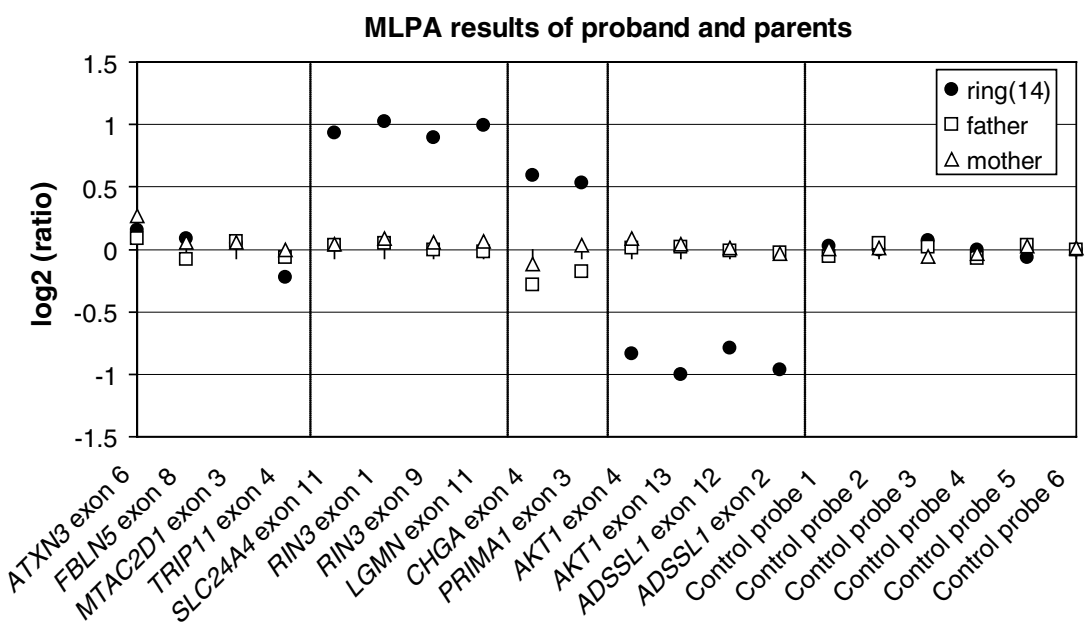

Figure 4 Log 2 ratio plot of the confirmatory MLPA. The proband (closed circles) shows triplication, duplication and deletion of the expected probes. Both parents show no copy number change in the tested regions. Three control probes were tested in duplo (control probes $1-6)$ and are located in the EXT1 gene on chromosome 8q24.11, in the CREBBP gene on $16 \mathrm{p} 13.3$ and in the $p 300$ gene on chromosome $22 \mathrm{q} 13.2$.

assume that an intrachromosomal U-type recombination during meiosis I, or a loop formation combined with one or two recombination events between homologous alleles, has occurred. The consequential recombinant dicentric chromosome would be deleted beyond the distal recombination site. At meiosis II, the two linked chromatids can segregate to the opposite poles resulting in a breakage between the two centromeres. If this breakage occurs asymmetrically, the two resulting recombinant products are a derivative with an inverted duplication and a deleted $14 \mathrm{q}$ derivative.
When chromosomal rearrangements take place, either constitutional or tumorigenic, broken chromosome ends need to be stabilized to prevent end-to-end fusions and exonucleolytic degradation. Telomere healing can be accomplished by addition of human telomeric tandem repeat sequence to broken chromosome ends ${ }^{20}$ or by telomere capture, which in fact is actually subtelomeric translocation to the broken chromosome end, resulting in an extra-duplicated subtelomeric region. This latter mechanism is proven in melanoma and other cancer cell lines and in irradiated lymphoblastoid and fibroblast cells. ${ }^{21}$ 
Examples of constitutional telomere capture are less common and is only reported a few times. ${ }^{22,23}$ Among the several inverted duplication/deletion events reported, ${ }^{7-9,15-19,23}$ in a single case the broken chromosome was proven to be repaired with telomere capture. ${ }^{23}$

On the contrary, in our case the healing of the broken end may have been mediated by ring formation. In an acrocentric chromosome, an additional break involving the short arm probably does not lead to an additional loss of coding sequence and consequently to impaired cell proliferation. The fact that ring chromosome formation in $47 \%$ of the reported cases ${ }^{6}$ involves acrocentric chromosomes, supports this notion.

If the recombination was based on non-allelic homologous recombination (NAHR), then a fragment with a normal copy number would still be present between two recombined low-copy repeats, between the duplicated and the deleted region at $14 \mathrm{q} 32.32$ to $14 \mathrm{q} 32.33$. As, neither the $1 \mathrm{Mb}$ BAC array nor the STR-allelotyping was conclusive to locate a possible fragment with a normal copy number between the duplicated and deleted region, an oligo array was performed. The 244k Agilent oligo array platform was chosen because between the distal duplicated and proximal deleted BAC clones there were 271 oligo reporters available, whereas, for example, in the $500 \mathrm{k}$ SNP-based chips from Affymetrix 120 SNP elements were present with an uneven coverage of the region of interest, including some gaps of $150 \mathrm{~kb}$.

Despite the fact that the oligo array did not detect a region of normal copy number between the duplicated and the deleted fragment, the region of the distal breakpoint could be narrowed down to $\sim 11 \mathrm{~kb}$.

Table 3 Comparison of the clinical features in published cases with distal chromosome 14 alterations and the presented case

\begin{tabular}{|c|c|c|c|c|c|c|c|c|}
\hline & \multicolumn{4}{|c|}{ Van Karnebeek et a ${ }^{25}$} & \multicolumn{4}{|c|}{ Chen et $a \rho^{\rho a}$} \\
\hline & \multicolumn{2}{|c|}{$\begin{array}{c}\text { Linear } 14 q \text { deletions } \\
\text { q32-distal (in } \%)\end{array}$} & \multicolumn{2}{|c|}{$\begin{array}{l}\text { Ring } 14 \text { deletions } \\
\text { q32-distal (in \%) }\end{array}$} & \multicolumn{2}{|c|}{$\begin{array}{l}14 q \text { duplications } \\
\text { q31-qter (in \%) }\end{array}$} & \multirow[t]{2}{*}{ Inv dup/del case } & \multirow[t]{2}{*}{$\begin{array}{l}\text { Our proband Inv } \\
\text { dup/del ring case }\end{array}$} \\
\hline No. of cases & 12 & & 20 & & 9 & & & \\
\hline Gender (males) & 3 & & 8 & & 5 & & & \\
\hline Mental retardation & $11 / 11$ & 100 & $20 / 20$ & 100 & $7 / 7$ & 100 & + & + \\
\hline Seizures & $1 / 10$ & 10 & $19 / 20$ & 95 & & & & + \\
\hline Hypotonia & $8 / 12$ & 67 & $11 / 19$ & 58 & $5 / 9$ & 56 & + & + \\
\hline Prenatal growth retardation & $5 / 12$ & 42 & $7 / 16$ & 44 & & & & + \\
\hline Post-natal growth retardation & $5 / 12$ & 42 & $9 / 17$ & 53 & $7 / 8$ & 88 & + & + \\
\hline Microcephaly & $4 / 12$ & 33 & $14 / 18$ & 78 & $6 / 9$ & 67 & + & + \\
\hline Dolichocephaly & $3 / 8$ & 38 & $5 / 12$ & 42 & & & & \\
\hline High forehead & $10 / 10$ & 100 & $7 / 12$ & 58 & & & & \\
\hline Prominent forehead & $7 / 10$ & 70 & $9 / 12$ & 75 & & & & \\
\hline Hypertelorism & $3 / 10$ & 30 & $7 / 15$ & 47 & $7 / 9$ & 78 & + & + \\
\hline Strabismus & $3 / 9$ & 33 & $4 / 15$ & 27 & $1 / 7$ & 14 & - & - \\
\hline Blepharophimosis & $6 / 10$ & 60 & $10 / 13$ & 77 & $0 / 7$ & 0 & + & + \\
\hline Ptosis & $4 / 11$ & 36 & $2 / 14$ & 14 & $0 / 7$ & 0 & - & - \\
\hline Downslanting palpebral fissures & $6 / 11$ & 55 & $8 / 13$ & 62 & $7 / 9$ & 78 & + & - \\
\hline Epi-/telecanthi & $8 / 12$ & 67 & $12 / 17$ & 71 & $2 / 9$ & 22 & + & - \\
\hline Broad/flat nasal bridge & $10 / 11$ & 91 & $10 / 13$ & 77 & & & & + \\
\hline Anteverted nares & $2 / 9$ & 22 & $5 / 12$ & 42 & & & & + \\
\hline Dysmorphic nose & & & & & $6 / 9$ & 67 & + & + \\
\hline Short bulbous nose & $5 / 10$ & 50 & $6 / 11$ & 55 & & & & - \\
\hline Long philtrum & $4 / 9$ & 44 & $4 / 11$ & 36 & & & & - \\
\hline Broad philtrum & $8 / 10$ & 80 & $8 / 11$ & 73 & & & & - \\
\hline Thin upper lip & $6 / 8$ & 75 & $5 / 11$ & 45 & & & & \\
\hline Small, fish-shaped mouth & $6 / 10$ & 60 & $6 / 13$ & 46 & & & & - \\
\hline Highly arched palate & 9/10 & 90 & $7 / 13$ & 54 & & & - & - \\
\hline Abnormal dentition & $2 / 6$ & 33 & $2 / 10$ & 20 & & & & \\
\hline Low set ears & $3 / 9$ & 33 & $7 / 8$ & 88 & & & & - \\
\hline Malformed helices & $5 / 8$ & 63 & $3 / 8$ & 38 & $7 / 9$ & 78 & + & + \\
\hline Micrognathia & $6 / 11$ & 55 & $5 / 12$ & 42 & $8 / 9$ & 89 & - & + \\
\hline Pointed chin & $4 / 10$ & 40 & $1 / 12$ & 8 & & & & - \\
\hline Short neck & $1 / 6$ & 17 & $7 / 14$ & 50 & & & & \\
\hline Webbed neck & $2 / 6$ & 33 & $1 / 11$ & 9 & & & & - \\
\hline Congenital heart defect & $3 / 9$ & 33 & $1 / 15$ & 7 & $6 / 9$ & 67 & + & + \\
\hline Single palmar crease & $6 / 7$ & 86 & $1 / 15$ & 7 & & & & + \\
\hline Brachydactyly & $0 / 3$ & 0 & $3 / 9$ & 33 & & & & - \\
\hline Clinodactyly & $2 / 7$ & 29 & $1 / 8$ & 13 & $7 / 9$ & 78 & + & - \\
\hline Tapering fingers/arachnodactyly & $2 / 5$ & 40 & $2 / 9$ & 22 & & & & \\
\hline Retinitis pigmentosa & $0 / 5$ & 0 & $8 / 16$ & 50 & & & & + \\
\hline Scoliosis & & & & & & & & + \\
\hline
\end{tabular}

\footnotetext{
${ }^{\mathrm{a} A n d}$ references herein.
} 
There was no evidence for the presence of LCRs in this interval (Ensembl, release 42), but this region does encompass a $2.8 \mathrm{~kb}$ chained self-alignment fragment having homology within its own region. Possibly this region can cause hairpin or loop formation and can mediate recombination or a double-strand break, eventually leading to the U-type of translocation.

Moreover, the oligo array showed an extra copy number gain of the first $600 \mathrm{~kb}$ of the duplicated region (Figure 3), at $14 \mathrm{q} 32.12$, which was confirmed with MLPA (Figure 4). Based on FISH results with a BAC probe from this region, it was found that the extra $600 \mathrm{~kb}$ fragment is inserted at the distal side of the chromosome, the part that is fused to the p-arm side of the centromere. In complex chromosome rearrangements, often more breaks are found than actually needed to explain the rearrangements on cytogenetic level. ${ }^{10,13,14}$ The extra amplification of this fragment at the distal side of the chromosome is probably a secondary event, following the inverted duplication and the distal break of the dicentric chromosome, illustrating the struggle to rescue the chromosome. The finding of this additional triplication stresses the importance of introducing high-resolution techniques in investigating genetic aberrations in patients.

Owing to the large overlap in the clinical features of cases with rearrangements within the distal region of chromosome 14 (Table 3), ring chromosome 14, 14q deletion and chromosome 14 distal duplication syndromes should be discussed together.

The ring chromosome 14 syndrome was delineated by Schmidt et $a l,{ }^{24}$ and it was further described by van Karnebeek et al. ${ }^{25}$ A distal duplication of chromosome 14 results in a variable clinical picture that is mainly depending on the size of the duplication. ${ }^{9,26}$ Main features that are found in distal duplications of $14 \mathrm{q} 31 \rightarrow$ qter are mental and growth retardation, microcephaly, hypertelorism, abnormal ears, micrognathia and congenital heart defects. In the case of Chen et al, an additional terminal deletion was demonstrated using FISH, next to the duplication. The small terminal deletion, which in itself can lead to a severe phenotype, ${ }^{25}$ or an additional duplication in a ring chromosome, is sometimes only detectable by molecular techniques such as FISH and arrayCGH. It emphasizes the significance of high-resolution molecular karyotyping for the establishment of accurate phenotype/genotype correlations.

\section{Acknowledgements}

We appreciate the supervision of E Aten in the MLPA analysis.

\section{References}

1 Riley SB, Buckton KE, Ratcliffe SG, Syme J: Inheritance of a ring 14 chromosome. J Med Genet 1981; 18: 209-213.
2 Donlan MA, Dolan CR: Ring chromosome 18 in a mother and son. Am J Med Genet 1986; 24: 171-174.

3 Fujimaki W, Baba K, Tatara K, Umezu R, Kusakawa S, Mashima Y: Ring chromosome 15 in a mother and her children. Hum Genet 1987; 76: 302

4 McKinlay Gardner RJ, Sutherland GR: Autosomal Ring Chromosomes in Chromosome Abnormalities and Genetic Counseling. New York: Oxford University Press, 2004, pp 178-185.

5 Sigurdardottir S, Goodman BK, Rutberg J, Thomas GH, Jabs EW, Geraghty MT: Clinical, cytogenetic, and fluorescence in situ hybridization findings in two cases of 'complete ring' syndrome. Am J Med Genet 1999; 87: 384-390.

6 Kosztolanyi G: Does 'ring syndrome' exist? An analysis of 207 case reports on patients with a ring autosome. Hum Genet 1987; 75: $174-179$.

7 Taylor KM, Francke U, Brown MG, George DL, Kaufhold M: Inverted tandem ('mirror') duplications in human chromosomes: inv dup 8p, 4q, 22q. Am J Med Genet 1977; 1: 3-19.

8 Weleber RG, Verma RS, Kimberling WJ, Fieger Jr HG, lubs HA: Duplication-deficiency of the short arm of chromosome 8 following artificial insemination. Ann Genet 1976; 19: 241-247.

9 Chen CP, Chern SR, Lin SP et al: A paternally derived inverted duplication of distal $14 \mathrm{q}$ with a terminal $14 \mathrm{q}$ deletion. Am J Med Genet Part A 2005; 139A: 146-150.

10 Knijnenburg J, Szuhai K, Giltay J et al: Insights from genomic microarrays into structural chromosome rearrangements. $A m ~ J$ Med Genet A 2005; 132: 36-40.

11 Fiegler H, Carr P, Douglas EJ et al: DNA microarrays for comparative genomic hybridization based on DOP-PCR amplification of BAC and PAC clones. Genes Chromosomes Cancer 2003; 36: $361-374$.

12 White SJ, Vink GR, Kriek M et al: Two-color multiplex ligationdependent probe amplification: detecting genomic rearrangements in hereditary multiple exostoses. Hum Mutat 2004; 24: $86-92$.

13 Gribble SM, Prigmore E, Burford DC et al: The complex nature of constitutional de novo apparently balanced translocations in patients presenting with abnormal phenotypes. J Med Genet 2005; 42: 8-16.

14 Rosenberg C, Knijnenburg J, Chauffaille ML et al: Array CGH detection of a cryptic deletion in a complex chromosome rearrangement. Hum Genet 2005; 116: 390-394.

15 Mewar R, Harrison W, Weaver DD, Palmer C, Davee MA, Overhauser J: Molecular cytogenetic determination of a deletion/duplication of $1 \mathrm{q}$ that results in a trisomy 18 syndrome-like phenotype. Am J Med Genet 1994; 52: 178-183.

16 Van Buggenhout G, Maas NM, Fryns JP, Vermeesch JR: A dysmorphic boy with 4qter deletion and 4q32.3-34.3 duplication: clinical, cytogenetic, and molecular findings. Am J Med Genet A 2004; 131: 186-189.

17 Genesio R, De Brasi D, Conti A et al: Inverted duplication of $15 \mathrm{q}$ with terminal deletion in a multiple malformed newborn with intrauterine growth failure and lethal phenotype. Am J Med Genet A 2004; 128: 422-428.

18 Bonaglia MC, Giorda R, Poggi G et al: Inverted duplications are recurrent rearrangements always associated with a distal deletion: description of a new case involving 2q. Eur J Hum Genet 2000; 8: 597-603.

19 Shimokawa O, Kurosawa K, Ida T et al: Molecular characterization of inv dup del(8p): analysis of five cases. Am J Med Genet A 2004; 128: $133-137$.

20 Wilkie AO, Lamb J, Harris PC, Finney RD, Higgs DR: A truncated human chromosome 16 associated with alpha thalassaemia is stabilized by addition of telomeric repeat (TTAGGG)n. Nature 1990; 346: 868-871.

21 Meltzer PS, Guan XY, Trent JM: Telomere capture stabilizes chromosome breakage. Nat Genet 1993; 4: 252-255.

22 Ballif BC, Kashork CD, Shaffer LG: FISHing for mechanisms of cytogenetically defined terminal deletions using chromosomespecific subtelomeric probes. Eur J Hum Genet 2000; 8: 764-770. 
23 Kostiner DR, Nguyen H, Cox VA, Cotter PD: Stabilization of a terminal inversion duplication of $8 \mathrm{p}$ by telomere capture from 18q. Cytogenet Genome Res 2002; 98: 9-12.

24 Schmidt R, Eviatar L, Nitowsky HM, Wong M, Miranda S: Ring chromosome 14 - a distinct clinical entity. J Medl Genet 1981; 18: 304-307.
25 van Karnebeek CDM, Quik S, Sluijter S, Hulsbeek MMF, Hoovers JMN, Hennekam RCM: Further delineation of the chromosome 14q terminal deletion syndrome. Am J Med Genet 2002; 110: $65-72$.

26 Carr DM, Jones-Quartey K, Vartanian MV, Moore-Kaplan H: Duplication 14(q31 $\rightarrow$ qter). J Med Genet 1987; 24: 372-374.

Supplementary Information accompanies the paper on European Journal of Human Genetics website (http://www.nature.com/ejhg) 